Check for updates

Cite this: Chem. Sci., 2019, 10, 4497

¿ All publication charges for this article have been paid for by the Royal Society of Chemistry

\section{Near-infrared aggregation-induced enhanced electrochemiluminescence from tetraphenylethylene nanocrystals: a new generation of ECL emitters $\dagger$}

\author{
Jia-Li Liu, Jia-Qi Zhang, Zhi-Ling Tang, Ying Zhuo, (D) Ya-Qin Chai (D)* \\ and Ruo Yuan (D) *
}

Herein, we observed near-infrared electrochemiluminescence (NIR ECL) emission from tetraphenylethylene nanocrystals (TPE NCs), which exhibit high ECL efficiency and excellent biocompatibility compared with the current NIR ECL emitters (such as semiconductor quantum dots and metal nanoclusters). The strong ECL signal of TPE NCs originates from the aggregation-induced enhanced ECL emission via improvement of the efficiency of electron hole recombination and suppression of the nonradiative transition. Impressively, the TPE NCs exhibit an enormous red-shifted $\mathrm{ECL}$ emission $(678 \mathrm{~nm}$ ) relative to the blue-light photoluminescence (PL) emission (440 nm). Compared to fluorescence imaging which is limited by photobleaching and autofluorescence, the NIR ECL emission of TPE NCs is highly favorable to diminish background interference over visible light and realize deeper tissue penetration, which expands the ECL emission of organic nanomaterials to the NIR region for broader biological applications.

Received 7th January 2019

Accepted 11th March 2019

DOI: $10.1039 / c 9 s c 00084 d$

rsc.li/chemical-science

\section{Introduction}

Near-infrared (NIR) emission, with the excellent merits of lower background interference, less photochemical damage, and deeper tissue penetration, has attracted continuous interest in biological sensing and imaging fields..$^{1,2}$ Noticeably, compared to NIR fluorescence, which is still faced with the inevitable limitations of autofluorescence and photobleaching in bioanalysis, electrochemiluminescence (ECL) with NIR emission has emerged as a powerful analysis tool in biomedical and diagnostic fields in terms of its versatility, high stability, nearzero background signal, and excellent controllability., Commonly, various types of semiconductor quantum dots (QDs) (CdTe, ${ }^{5,6} \mathrm{CdSeTe} / \mathrm{ZnS},{ }^{7} \mathrm{PbS},{ }^{8}$ etc.) as traditional NIR ECL emitters have been intensively investigated, while heavy metalcontaining QDs were highly biotoxic causing severe health concerns. ${ }^{9,10}$ To explore the ECL emission in the NIR region with low toxicity components, noble metal nanoclusters (NCs) ${ }^{11}$ consisting of a few to dozens of atoms are significantly attractive in bioanalysis applications. Wang and co-workers synthesized lipoic acid stabilized $\mathrm{Au}_{22} \mathrm{NCs}$ covalently cross-linked with $\mathrm{N}, \mathrm{N}$ diethylethylenediamine as the coreactant and reported the NIR

Key Laboratory of Luminescent and Real-Time Analytical Chemistry, Ministry of Education, College of Chemistry and Chemical Engineering, Southwest University, Chongqing 400715, China.E-mail: yqchai@swu.edu.cn; yuanruo@swu.edu.cn

$\dagger$ Electronic supplementary information (ESI) available: Reagents and materials, apparatus, and Fig. S1-S4. See DOI: 10.1039/c9sc00084d
ECL of aqueous soluble AuNCs. ${ }^{12}$ Despite the remarkable progress of the proposed NIR ECL emitters, the ECL efficiency of semiconductor QDs and metal NCs was still restricted. Lately, organic materials with specific performances of flexible preparation and low toxicity have been proposed for application in biological fluorescence imaging, ${ }^{13,14}$ organic lightemitting diodes (OLEDs) ${ }^{15}$ and organic field-effect transistors. ${ }^{16}$ Significantly, the exploration of ECL emissions of organic nanoparticles (NPs) ${ }^{17,18}$ has experienced increasing attention and progress. Bard and co-workers investigated a series of organic NPs (such as polyaromatic compounds and their derivatives, ${ }^{19}$ and organic polymers ${ }^{20,21}$ ) and were dedicated to improving the ECL efficiency of organic NPs as good candidates for ECL devices. However, the poor biocompatibility and blue-light ECL emission of the above organic NPs limited their further applications in ECL sensing and bioimaging. In view of these deficiencies, it is of critical significance to develop highly efficient ECL emitters with NIR ECL properties and low biotoxicity.

Aggregation-induced emission (AIE), an effective fluorescence enhancement strategy, has potential applications in optoelectronic devices and cell imaging, because strong FL emission was found when individual organic molecules aggregated together, while the individual ones in dilute solutions were non-emissive. ${ }^{22}$ More recently, Carrara et al. pioneered the aggregation-induced ECL properties of squareplanar Pt(II) complexes, which paved a new way to improve 
the ECL efficiency of the next generation of brilliant emitters. ${ }^{23}$ However, noble metal complexes as ECL emitters not only increase the experimental cost, but also show biotoxicity and poor biocompatibility. It is essential to develop metal-free ECL emitters with aggregation-induced enhanced ECL properties for ECL biosensing and imaging applications. In this paper, we synthesized tetraphenylethylene nanocrystals (TPE NCs) and observed the aggregation-induced enhanced NIR ECL emission phenomenon in aqueous solution, while the ECL signal of individual TPE molecules is basically invisible in organic solvents (Scheme 1A). The strong ECL emission of TPE NCs is ascribed to two possible reasons. Firstly, energy gaps were reduced when the individual TPE molecules aggregated together to form TPE NCs, which achieved high efficiency of electron hole recombination to obtain the excited state via the ECL route. ${ }^{24}$ Moreover, the intramolecular free rotation of phenyl rings in the TPE NCs was restricted after the aggregation, which reduced the non-radiative relaxation to realize further ECL enhancement. Interestingly, compared to the maximum emission peak of the PL spectra (440 nm) and EL spectra (442 nm) from TPE NCs, the ECL emission peak of TPE NCs at $678 \mathrm{~nm}$ was observed, which expanded the study on the aggregation-induced ECL emission of organic NPs to the NIR region. The NIR ECL emission mechanism of TPE NCs may be correlated with the high conjugation degree of the aggregation of TPE molecules in TPE NCs and the narrow energy gaps of the surface states of TPE NCs via the ECL route, which distinguished from the band gap transition of PL. ${ }^{25}$ Furthermore, compared with conventional organic ECL emitters, the TPE NCs exhibit favorable biocompatibility because bovine serum albumin (BSA) with abundant amino acid residues was employed as the capping agent. The preparation and ECL properties of TPE NCs are exhibited in Scheme 1. Initially, the TPE NCs were synthesized from individual TPE by selfassembly. Meanwhile, BSA was wrapped on the TPE NCs to offer a hydrophilic surface (part A). Impressively, as shown in part B, the TPE NCs were covered on the electrode surface to

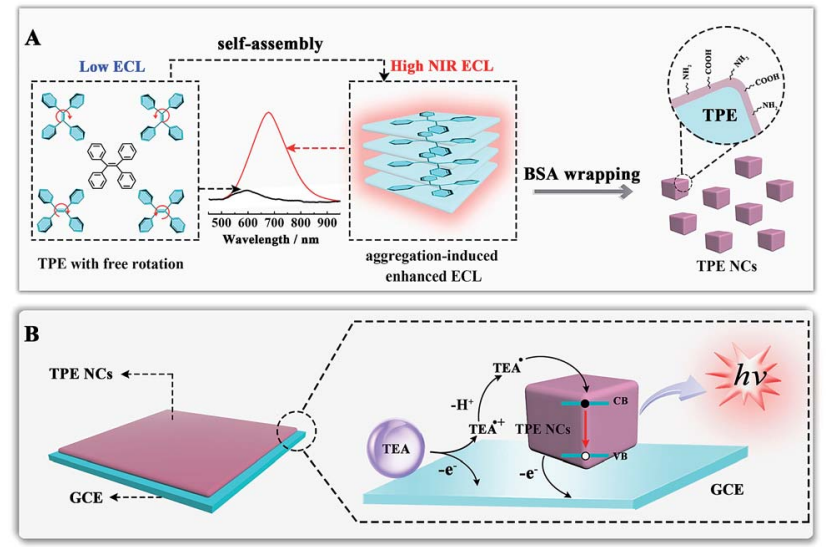

Scheme 1 (A) Schematic illustration of the preparation of TPE NCs by self-assembly and the NIR aggregation-induced enhanced ECL properties; (B) illustration of the possible ECL mechanism of the TPE/ TEA system. afford an intense ECL signal with the coreactant of triethylamine (TEA). Inspired by the intense NIR ECL emission and good biocompatibility, the TPE NCs as a new generation of ECL emitters are expected to show great promise in ECL sensing and bioimaging.

\section{Experimental section}

\section{Preparation of TPE nanocrystals (NCs)}

The TPE nanocrystals (NCs) were prepared via the desolvation method. Briefly, TPE powder $(0.5 \mathrm{mg})$ was dissolved in $0.5 \mathrm{~mL}$ of tetrahydrofuran (THF) to obtain a homogeneous colorless TPE solution ( $1 \mathrm{mg} \mathrm{mL}{ }^{-1}$ ). Subsequently, $5 \mathrm{~mL}$ of deionized water was used to disperse $10 \mathrm{mg}$ of BSA under sonication at room temperature. Then, $0.5 \mathrm{~mL}$ of TPE solution was slowly injected into the BSA solution under sonication, followed by stirring at room temperature for 2 hours. Ultimately, after removing the THF and washing with deionized water, the TPE NCs were obtained by vacuum desiccation.

\section{Results and discussion}

\section{Characterization of TPE nanocrystals (NCs)}

The morphology of TPE nanocrystals (NCs) was investigated. As depicted in Fig. 1A, the SEM results indicate that TPE NCs with an average size of $450 \mathrm{~nm} \pm 50 \mathrm{~nm}$ are clearly observed. Meanwhile, the TPE NCs presented homogeneous diameters within a larger field of vision. Additionally, TEM and AFM were also performed to characterize the features of TPE NCs. As shown in Fig. 1B and C, both the images of TPE NCs reveal nanoblock structures, which conformed to the SEM results. To further investigate the particle size distribution, dynamic light scattering (DLS) was employed to analyze the TPE NCs. As illustrated in Fig. 1D, TPE NCs are well distributed with an average size of $500 \mathrm{~nm} \pm 100 \mathrm{~nm}$, and are slightly bigger than the size of TPE NCs in SEM images, attributed to the hydrogen bonding between the amidogen of TPE NCs and $\mathrm{H}_{2} \mathrm{O}$.
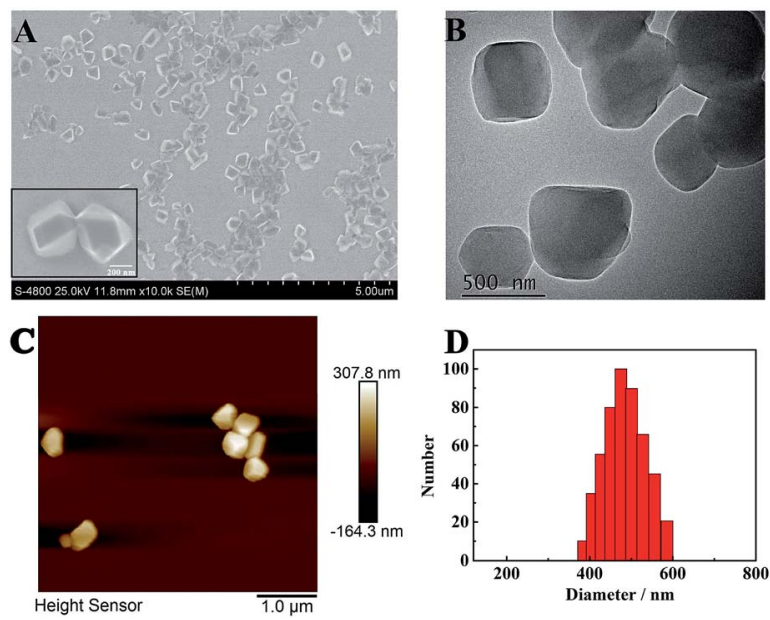

Fig. 1 (A) SEM, (B) TEM, and (C) AFM images, and (D) size distribution of the TPE NCS. 
Furthermore, the crystallographic structure of TPE NCs was confirmed to be monoclinic by X-ray diffraction (XRD). As displayed in Fig. 2A, the peaks of TPE NCs (curve a) perfectly correspond to the peak of the standard PDF card (curve b) of the TPE crystal (CCDC code TPHETY), which is consistent with the previous literature. ${ }^{26}$ Meanwhile, the predicted structure of TPE NCs is simulated in Fig. 2B, and the formation of an ordered monoclinic crystal structure is attributed to the aromatic stacking interactions among the TPE molecules (Fig. 2C).

\section{Spectral characteristics of the TPE NCs}

The optical properties of TPE NCs were measured by UV-vis absorption, photoluminescence (PL) and ECL spectra, respectively. As depicted in Fig. S1, $\dagger$ a distinct absorption peak of TPE dissolved in tetrahydrofuran (THF) is observed at $308 \mathrm{~nm}$ (curve a). However, the UV-vis absorption peak of TPE NCs (curve b) is red-shifted to $380 \mathrm{~nm}$, which indicates that the TPE NCs aggregated in solution with the help of BSA. As depicted in Fig. 3A, the PL excitation peaks of TPE NCs are at $371 \mathrm{~nm}$ and $387 \mathrm{~nm}$, and the maximum PL emission peak is located at $440 \mathrm{~nm}$. Under the excitation light of $488 \mathrm{~nm}$, the TPE NCs emitted strong blue fluorescence, and the morphology and size of TPE NCs in the fluorescence microscopic image (Fig. 3B) were in accordance with those of TPE NCs in the TEM image. Interestingly, the ECL spectra of individual TPE molecules in acetonitrile by spectral accumulation displayed an ECL emission peak $(600 \mathrm{~nm})$ and a weak shoulder ECL peak $(440 \mathrm{~nm})$ which is in accordance with the PL spectra (Fig. 3C), originating from the dimers and monomers of the TPE molecule, respectively. ${ }^{27}$ However, an intense NIR ECL emission peak $(678 \mathrm{~nm})$ of the TPE NCs was obtained
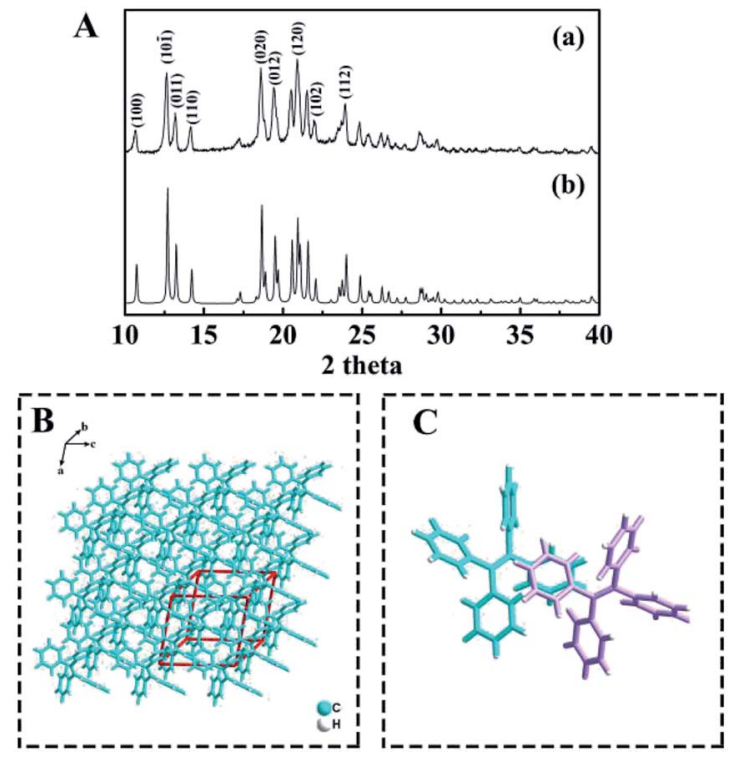

Fig. 2 (A) XRD patterns of (a) the TPE NCs and (b) the standard PDF card of the TPE crystal, (B) the predicted morphology of TPE crystals, and $(C)$ the predicted interaction between the two TPE molecules represented in different colors.
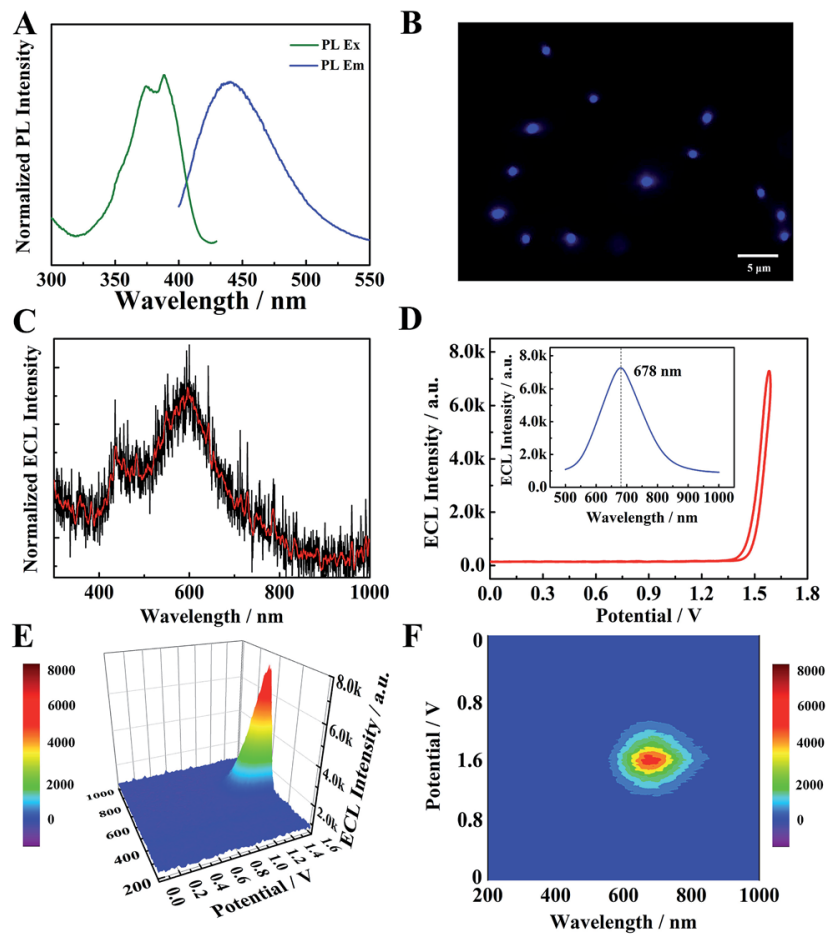

Fig. 3 (A) Normalized PL excitation spectra and PL emission spectra of TPE NCs in PBS solution. (B) Fluorescence microscopy image of TPE NCs. (C) The ECL spectra of individual TPE molecules in acetonitrile with TEA as a coreactant. (D) The ECL-potential curve of TPE NCs. The inset of (D) the 2D ECL spectra of TPE NCs in PBS solution with TEA as a coreactant. The ECL-potential-wavelength of TPE NCs via a 3D surface image (E) and 3D heat map image (F).

in the presence of TEA via ECL spectral analysis (Fig. 3D-F), exhibiting a significant red shift $(\Delta \lambda=238 \mathrm{~nm})$ compared with the PL spectra. In addition, the electroluminescence (EL) emission peak of the TPE film (Fig. S2 $\dagger$ ) was measured at $442 \mathrm{~nm}$ (basically consistent with the PL spectra) with an exciplex emission of $492 \mathrm{~nm}$, which demonstrated that both the luminescence mechanisms of PL and EL are different from that of ECL in TPE NCs. In order to further investigate the large red shift of ECL spectra, the energy levels of TPE NCs were evaluated using the cyclic voltammogram (CV) of the TPE NCs modified GCE in $0.1 \mathrm{M} \mathrm{Bu}_{4} \mathrm{NBF}_{4}$ acetonitrile solution bubbled with $\mathrm{N}_{2}$ for $30 \mathrm{~min}$ (Fig. S3A $\dagger$ ). During the anodic scan, an oxidation wave with an onset potential of $0.995 \mathrm{~V} v s . \mathrm{Ag} / \mathrm{Ag}^{+}$ was obtained, while the reduction wave was detected at $-0.705 \mathrm{~V}$. Accordingly, the valence band (VB) and conduction band (CB) were calculated to be $-5.40 \mathrm{eV}$ and $-3.54 \mathrm{eV}$, respectively, and the energy gap was estimated to be approximately $1.86 \mathrm{eV}$, which is far less than the energy gap (3.04 eV) calculated from the lowest exciton absorption peak of TPE NCs at $408 \mathrm{~nm}$ by UV-vis diffuse reflectance spectra (DRS) (Fig. S3B $\dagger$ ). Simultaneously, the emission state energy of TPE NCs was calculated to be $2.82 \mathrm{eV}$ from the PL spectra by $E_{\mathrm{S}}(\mathrm{eV})$ $=1240 / \lambda(\mathrm{nm})$, which is very approximate to the optical energy gap $(3.04 \mathrm{eV})$. It is speculated that the electron injected into the surface trap of TPE $\mathrm{NCs}^{28}$ results in a relatively small 
energy gap via the ECL route to produce a red-shifted wavelength, which is different from the PL mechanisms originating from the nuclear band gap pathway. Therefore, the NIR ECL mechanism of TPE NCs was attributed to the higher conjugation degree of the aggregation of TPE molecules in TPE NCs and the surface states of TPE NCs via the ECL route.

Furthermore, to study the aggregation-induced ECL enhancement mechanism, the cyclic voltammograms (CV) and ECL of individual TPE molecules and TPE NCs in acetonitrile were recorded, respectively. As depicted in Fig. 4A, the individual TPE molecules exhibit two extremely weak ECL emission peaks at $-1 \mathrm{~V}$ and $1.6 \mathrm{~V}$, for the free TPE molecules in acetonitrile had a wide energy gap which made the excited state unobtainable via the ECL route. However, the two corresponding ECL emission peaks of the TPE NCs in acetonitrile zoomed to approximately 4000 a.u (Fig. 4B) which was 20 -fold higher than that of the free TPE molecules. Comparing the ECL intensity of single TPE molecules with that of TPE NCs, the aggregated TPE molecules in TPE NCs showed an intense ECL signal, which was attributed to the following causes. On the one hand, the reduced energy gaps in the ordered crystal structure made the excited state easily obtainable via the ECL route. On the other hand, the intramolecular motions of phenyl rings in the TPE NCs were limited inhibiting the non-radiative channels and realizing the radiative transition for further ECL enhancement. Interestingly, a relatively strong anodic ECL signal of TPE NCs in PBS was obtained from $-2-1.6 \mathrm{~V}$ without cathodic ECL emission (Fig. S4 $\dagger$ ), which provided evidence that the radical cation of TPE $\left(\mathrm{TPE}^{+}\right)$was more stable than the radical anion. In consequence, we chose the anode ECL signal of the TPE NCs for further study. Moreover, the $\left[\mathrm{Ru}(\mathrm{bpy})_{3}\right]^{2+} /$ TPA system was selected as the standard to calculate the relative ECL efficiency of the TPE NCs/TPA system (the detailed formula is shown in the ESI $\dagger$ ) and it was found to be $1.35 \%$, which was higher than those of polyaniline- $\left[\mathrm{Ru}(\mathrm{bpy})_{2}\right.$ $\mathrm{PIC}]^{2+}(1.0 \%)^{29}$ and Mn@CdInS films $(0.8 \%) .^{30}$

\section{Possible ECL mechanism of the TPE/TEA system}

To identify the ECL mechanism of the TPE/TEA system, correlative DPV and ECL curves are shown in Fig. 5. Firstly, when the TPE NCs modified GCE was measured in PBS, the DPV showed inconspicuous oxidation and reduction peaks (curve a and curve $\mathrm{a}^{\prime}$, Fig. $5 \mathrm{~A}$ ). Meanwhile, the ECL signal with approximately 910 a.u. could be observed (curve a, Fig. 5B). However, an oxidation peak appeared and both the redox currents from 0.6 to $1.6 \mathrm{~V}$ were significantly
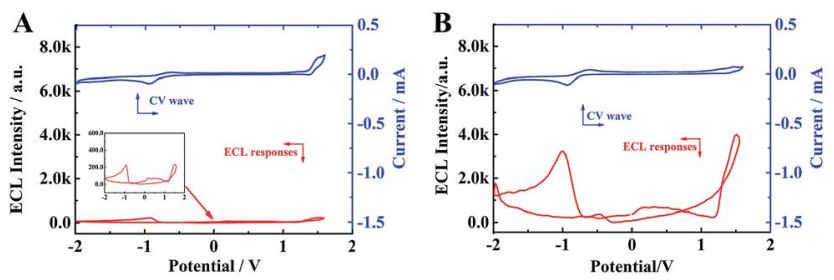

Fig. 4 CV curves (blue line) and the corresponding ECL-potential profiles (red line) of (A) $2 \mathrm{mM}$ TPE and (B) TPE NCs in $0.1 \mathrm{M} \mathrm{Bu}_{4} \mathrm{NBF}_{4}$ acetonitrile solution.
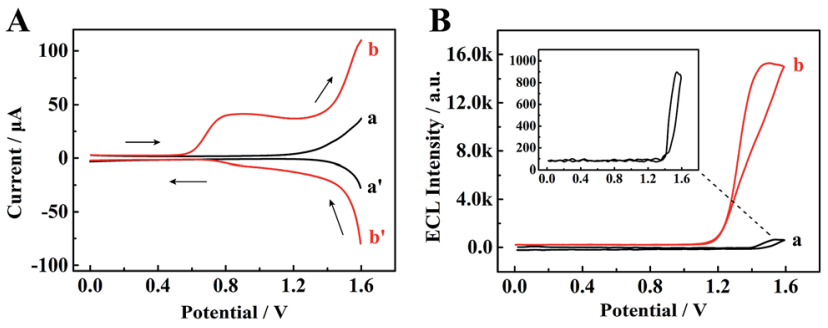

Fig. 5 (A) Differential pulse voltammetry (DPV) and (B) ECL-potential profiles of the TPE NCs modified GCE in (a) PBS solution and (b) PBS + 18 mM TEA solution.

enhanced (curve $\mathrm{b}$ and curve $\mathrm{b}^{\prime}$, Fig. 5A) after adding TEA as the coreactant into PBS, which was attributed to the generation of the TEA radical cation $\left(\mathrm{TEA}^{+}\right)$for further dehydrogenation to form a strong reductant $\mathrm{TEA}^{\circ}(\sim-1.7 \mathrm{~V} v$ s. $\mathrm{Ag} / \mathrm{AgCl}) .{ }^{31}$ Subsequently, an electron from TEA $^{\circ}$ was transferred to the deep surface traps of TPE NCs with lower energies to achieve radiative recombination between the electrons and holes at the surface states of TPE NCs resulting in the excited state of TPE (TPE*). When the TPE* transited back to the ground state, an intense ECL emission was achieved, which was 16.8 -fold higher than that of the TPE NCs modified GCE detected in individual PBS (curve b, Fig. 5B). The possible ECL mechanisms of the TPE/TEA system are depicted as follows:

$$
\begin{gathered}
\mathrm{TPE}-\mathrm{e}^{-} \rightarrow \mathrm{TPE}^{+} \\
\mathrm{TEA}-\mathrm{e}^{-} \rightarrow \mathrm{TEA}^{++} \\
\mathrm{TEA}^{++} \rightarrow \mathrm{TEA}^{\cdot}+\mathrm{H}^{+} \\
\mathrm{TPE}^{+}+\mathrm{TEA}^{\cdot} \rightarrow \mathrm{TPE}^{*}+\text { product } \\
\mathrm{TPE}^{*} \rightarrow \mathrm{TPE}+h v
\end{gathered}
$$

\section{Conclusions}

In summary, unlike the low ECL efficiency and poor biocompatibility of traditional NIR ECL emitters, tetraphenylethylene nanocrystals (TPE NCs) offer an intense NIR ECL signal thanks to the aggregation-induced enhanced ECL emission via improvement of the efficiency of electron hole recombination and suppression of the nonradiative transition. Impressively, compared with the blue-light photoluminescence (PL) emission $(440 \mathrm{~nm})$, the TPE NCs show strong ECL emission $(678 \mathrm{~nm})$ in the NIR region, and hold great promise in ECL sensing and bioimaging due to lower background interference over visible light and deeper tissue penetration. Notably, the described TPE NCs reveal a new avenue to explore highly efficient ECL emitters for biological applications.

\section{Conflicts of interest}

There are no conflicts to declare. 


\section{Acknowledgements}

This work was financially supported by the NNSF of China (21775124, 21575116, 21675129, and 51473136), and the Fundamental Research Funds for the Central Universities (XDJK2018AA003), China.

\section{References}

1 T. Rauch, M. Böberl, S. F. Tedde, J. Fürst, M. V. Kovalenko, G. Hesser, U. Lemmer, W. G. Heiss and O. Hayden, Nat. Photonics, 2009, 3, 332-336.

2 T. A. Redchuk, A. A. Kaberniuk and V. V. Verkhusha, Nat. Protoc., 2018, 13, 1121-1136.

3 K. N. Swanick, M. Hesari, M. S. Workentin and Z. F. Ding, J. Am. Chem. Soc., 2012, 134, 15205-15208.

4 S. Voci, B. Goudeau, G. Valenti, A. Lesch, M. Jovic, S. Rapino, F. Paolucci, S. Arbault and N. Sojic, J. Am. Chem. Soc., 2018, 140, 14753-14760.

5 N. Gaponik, I. L. Radtchenko, G. B. Sukhorukov, H. Weller and A. L. Rogach, Adv. Mater., 2002, 14, 879-882.

6 Y. P. He, F. Zhang, B. Zhang and G. Z. Zou, Anal. Chem., 2018, 90, 5474-5480.

7 A. J. Stewart, K. Brown and L. Dennany, Anal. Chem., 2018, 90, 12944-12950.

8 M. Hesari, K. N. Swanick, J. S. Lu, R. Whyte, S. Wang and Z. F. Ding, J. Am. Chem. Soc., 2015, 137, 11266-11269.

9 A. M. Derfus, W. C. W. Chan and S. N. Bhatia, Nano Lett., 2004, 4, 11-18.

10 K. Wu, Y. S. Park, J. Lim and V. I Klimov, Nat. Nanotechnol., 2017, 12, 1140-1147.

11 M. Hesari, M. S. Workentin and Z. F. Ding, Chem. Sci., 2014, 5, 3814-3822.

12 T. Y. Wang, D. C. Wang, J. W. Padelford, J. Jiang and G. L. Wang, J. Am. Chem. Soc., 2016, 138, 6380-6383.

13 H. Wan, J. Y. Yue, S. J. Zhu, T. Uno, X. D. Zhang, Q. L. Yang, K. Yu, G. S. Hong, J. Y. Wang, L. L. Li, Z. R. Ma, H. P Gao, Y. T. Zhong, J. Su, A. L. Antaris, Y. Xia, J. Luo, Y. Y. Liang and H. J Dai, Nat. Commun., 2018, 9, 1171.

14 Q. Miao and K. Pu, Adv. Mater., 2018, 30, 1801778.
15 W. X. Zeng, H. Y. Lai, W. K. Lee, M. Jiao, Y. J. Shiu, C. Zhong, S. L. Gong, T. Zhou, G. H. Xie, M. Sarma, K. T. Wong, C. C. Wu and C. L. Yang, Adv. Mater., 2018, 30, 1704961.

16 A. Lv, M. Freitag, K. M. Chepiga, A. H. Schäfer, F. Glorius and L. F. Chi, Angew. Chem., Int. Ed., 2018, 57, 4792-4796.

17 D. O'Carroll, D. Iacopino, A. O'Riordan, P. Lovera, É. O'Connor, G. A. O'Brien and G. Redmond, Adv. Mater., 2008, 20, 42-48.

18 F. Rizzo, F. Polo, G. Bottaro, S. Fantacci, S. Antonello, L. Armelao, S. Quici and F. Maran, J. Am. Chem. Soc., 2017, 139, 2060-2069.

19 K. M. Omer and A. J. Bard, J. Phys. Chem. C, 2009, 113, 1157511578.

20 J. Suk, Z. Wu, L. Wang and A. J. Bard, J. Am. Chem. Soc., 2011, 133, 14675-14685.

21 Y. L. Chang, R. E. Palacios, F. R. F. Fan, A. J. Bard and P. F. Barbara, J. Am. Chem. Soc., 2008, 130, 8906-8907.

22 Y. Hong, J. W. Lam and B. Z. Tang, Chem. Soc. Rev., 2011, 40, 5361-5388.

23 S. Carrara, A. Aliprandi, C. F. Hogan and L. De Cola, J. Am. Chem. Soc., 2017, 139, 14605-14610.

24 S. L. Liu, Q. H. Zhang, L. Zhang, L. Gu, G. Z. Zou, J. C. Bao and Z. H. Dai, J. Am. Chem. Soc., 2016, 138, 1154-1157.

25 S. Liu, Q. H. Zhang, L. Zhang, L. Gu, G. Z. Zou, J. C. Bao and Z. H. Dai, J. Am. Chem. Soc., 2016, 138, 1154-1157.

26 I. Ino, L. P. Wu, M. Munakata, T. Kuroda-Sowa, M. Maekawa, Y. Suenaga and R. Sakai, Inorg. Chem., 2000, 39, 5430-5436.

27 E. F. Fabrizio, I. Prieto and A. J. Bard, J. Am. Chem. Soc., 2000, 122, 4996-4997.

28 S. L. Liu, Q. Q. Zhang, L. Gu, L. Zhang, G. Z. Zou, J. C. Bao and Z. H. Dai, J. Am. Chem. Soc., 2016, 138, 1154-1157.

29 K. M. Molapo, A. Venkatanarayanan, C. M. Dolan, U. Prendergast, P. G. Baker, E. I. Iwuoha, T. E. Keyes and R. J. Forster, Electrochem. Commun., 2014, 48, 95-98.

30 F. Wang, J. Lin, T. Zhao, D. Hu, T. Wu and Y. Liu, J. Am. Chem. Soc., 2016, 138, 7718-7724.

31 R. Y. Lai and A. J. Bard, J. Phys. Chem. A, 2003, 107, 33353340 . 